\title{
Optimality of beamforming condition for multiple antenna systems with mean feedback
}

Wen Zhou ${ }^{1 *}$, Hongyang Chen ${ }^{2}$ and Wong Hing Lam

\begin{abstract}
The necessary and sufficient condition for beamforming optimality for multiple-input multiple output (MIMO) systems with mean feedback has been derived and thoroughly investigated in this article. The condition is an inequality that contains parameters of the number of antennas, transmission power, and the singular values of channel mean matrix. When the beamforming optimality condition is satisfied, the Shannon capacity of MIMO system can be achieved by beamforming and hence scalar codes can therefore be used to achieve the maximum capacity. The channel is modeled as the sum of a scattering matrix of i.i.d zero mean, unit variance complex Gaussian random variables, and a mean matrix having its rank $r \geq 1$. The beamforming condition inequality performance versus system parameters of signal-to-noise ratio and Ricean factor have thoroughly been investigated by numerical results. Computer simulations show that the beamforming strategy can be adopted on a relatively relaxed beamforming condition without the loss of capacity performance.
\end{abstract}

Keywords: Multiple-input multiple output (MIMO), beamforming, mean feedback, rank

\section{Introduction}

In the long-term evolution advanced (LTE-A) [1] of the 3rd Generation Partnership Project, multiple-input multiple output (MIMO) has been considered as a key technology in the physical layer. Telatar [2] and Foschini [3] first formulated the system capacity of the MIMO systems assuming independent and identically distributed fading at different antennas. Having acquired the perfect channel state information (CSI) in the MIMO systems, the transmitter performs a linear transform by multiplying the eigenvector of channel matrix and allocating the power of each data stream by waterfilling algorithm, its system capacity can be obtained. When the transmitter has only partial CSI such as the statistical information of the channel, acquiring the optimal transmission covariance matrix and deriving the condition for beamforming optimality are two major obstacles. A few studies on this aspect have been presented [4-11]. For one issue, to achieve maximum system capacity, the optimal transmission of covariance matrix is essential to the optimal transmitter. For another, by verifying whether the beamforming optimality condition is satisfied, we can determine whether the transmitter

\footnotetext{
* Correspondence: wzhou@stu.edu.cn

'Department of Electronic Engineering, Shantou University, Shantou, China Full list of author information is available at the end of the article
}

can adopt the beamforming strategy. If so, the operation at the transmitter will be simplified significantly since exhaustive searching for the optimal power allocation is no longer needed and therefore the scalar codes instead of vector codes can be adopted.

Usually, there are two kinds of feedback mechanisms, namely, the mean and the covariance feedbacks [4,6-10]. Visotsky and Madhow [4] first resolved the transmitter optimization and derived the optimality condition for beamforming under two cases of mean and covariance feedbacks for the consideration of the multiple-input single-output system. Later, Jafar and Goldsmith [6] extended Visotsky and Madhow's work to MIMO systems, with the assumption that the rank of channel mean matrix is one for channel mean feedback. In [8], the optimal transmission directions and the condition for beamforming optimality have been derived to maximize the effective capacity. The concept of effective capacity [12] was proposed to measure the quality of service. Jorswieck et al. [8] mainly focused on the case of covariance and the mean feedbacks were not considered. Li et al. [10] studied the transmitter optimization and beamforming optimality conditions for double-scattering MIMO channels. The channel model was characterized as a matrix product of transmitter, 
receiver, and scattering correlation matrices and the covariance feedback was used.

This paper focuses on the derivation of beamforming optimality condition under the mean feedback. In general, the rank of the covariance matrix of the transmission signal is larger than one. By limiting the covariance matrix of the transmitting signal to be one, all power at the transmitter is allocated to a single data stream and therefore the MIMO system is reduced to a single-input single-output system. Correspondingly, this kind of transmission strategy is termed as beamforming strategy. Usually, the rank of optimal transmission covariance matrix of achieving the maximum system capacity is not equal to 1 , i.e., the beamforming strategy is not necessarily the optimum strategy. Only if the system settings satisfy a certain condition, then the beamforming strategy achieves the maximum system capacity and it is optimal. Corresponding condition is referred to as the beamforming optimality condition. In this study, the rank of channel mean matrix is generalized and it is not limited to be 1. Therefore, this study is an extension of [6]. The condition for the beamforming optimality is an inequality that contains parameters such as the number of antennas, transmission power, and the singular values of channel mean matrix. The inequality is derived and also thoroughly investigated by numerical method. We also compare the ergodic capacities of the beamforming strategy, optimum strategy, and the equal power allocation method. We also provide a list of conditions under which the beamforming strategy can be adopted.

The rest of the paper is organized as follows. Section 2 describes the MIMO system model. Section 3 derives the necessary and sufficient condition for beamforming optimality under mean feedback. The ergodic capacities for beamforming and optimum strategies have been presented in Section 4. Numerical results and computer simulation are discussed in Section 5, followed by conclusion in Section 6.

\section{System model}

Consider the multiple antenna systems equipped with $N_{T}$ transmitter antennas and $N_{R}$ receiver antennas. Assuming that the MIMO channel is flat fading, the received signal is given by

$$
\mathrm{y}=\mathbf{H x}+\mathbf{w},
$$

where $\mathbf{x} \in \mathbb{C}^{N_{T}}$ is the transmitted signal, $\mathbf{y} \in \mathbb{C}^{N_{R}}$ is the received signal, $\mathbf{H} \in \mathbb{C}^{N_{R} \times N_{T}}$ is the channel matrix, $\mathbb{C}$ stands for the complex number field, $\mathbf{w} \in \mathbb{C}^{N_{R}}$ is the complex additive white Gaussian noise vector having i.i. $\mathrm{d}$ elements with zero means and $\sigma^{2}$ variances. The transmitted signal $\mathbf{x}$ satisfies a power constraint, $E\left(\mathbf{X}^{T} \mathbf{X}\right)$ $\leq P$, where $E(\cdot)$ denotes the expectation operation and (.)
${ }^{T}$ stands for conjugate. The channel matrix $\mathbf{H}$ is normalized and expressed by

$$
\begin{aligned}
\mathbf{H} & =A_{1} \mathbf{H}_{\mu}+A_{2} \mathbf{H}_{\mathbf{w}} \\
& =\sqrt{\frac{K}{K+1}} \mathbf{H}_{\mu}+\sqrt{\frac{1}{K+1}} \mathbf{H}_{\mathbf{w}}
\end{aligned}
$$

where $K$ is the Ricean factor. The matrix $\mathbf{H}_{\boldsymbol{\mu}}$ is a deterministic mean matrix which represents the LOS component and satisfies

$$
\operatorname{Tr}\left(\mathbf{H}_{\mu} \mathbf{H}_{\mu}^{H}\right)=N_{R} N_{T}
$$

where $\operatorname{Tr}(\cdot)$ denotes the trace operation, $(\cdot)^{H}$ stands for the Hermitian operation. The matrix $\mathbf{H}_{\mathbf{w}}$ is a complex Gaussian matrix representing scattering component, which has independent circularly complex Gaussian elements with zero means and unit variances. It is assumed that the receiver has perfect each realization of $\mathbf{H}$ and the transmitter has only the mean information $\mathbf{H}_{\boldsymbol{\mu}}$ and Ricean factor $K$. The input distribution that maximizes the mutual information is always circularly complex Gaussian [2]. The MIMO system capacity is therefore given by

$$
C=\max _{Q: \operatorname{Tr}(Q) \leq P} C(Q),
$$

where

$$
C(Q)=E\left(\log \left|I_{N_{R}}+\frac{H Q H^{H}}{\sigma^{2}}\right|\right),
$$

$Q$ is the transmission covariance matrix. The MIMO system signal-to-noise ratio (SNR) is defined as the SNR at each receiver antenna and it is given by

$$
\mathrm{SNR}=\frac{P}{\sigma^{2}}
$$

\section{The condition for beamforming optimality under mean feedback}

The necessary and sufficient condition for optimality of beamforming for MIMO systems under mean feedback is derived in Section 3.1. The issue about numerical evaluation for the condition is also addressed in Section 3.2.

\subsection{Derivation of beamforming optimality condition}

We start with the results presented in [13]. Venkatesan et al. [13] have proved that the optimal transmit covariance $Q$ has the same eigenvectors as the channel mean matrix $\mathbf{H}_{\boldsymbol{\mu}}$. Let

$$
\mathbf{H}_{\mu}=U_{\mu} \Sigma_{\mu} V_{\mu}^{H}
$$


be the singular value decomposition (SVD) of channel mean matrix $\mathbf{H}_{\boldsymbol{\mu}}$, and

$$
Q=U_{Q} \Sigma_{Q} U_{Q}^{H}
$$

be the spectral decomposition of $Q$. We have

$$
U_{Q}=V_{\mu} \text {. }
$$

Substituting (7)-(9) into (5) and noting that the statistics of matrix after multiplication of $\mathbf{H}_{\mathbf{w}}$ with a unitary matrix remains unchanged, we have

$$
C=\max _{\Sigma_{Q}: \operatorname{Tr}\left(\Sigma_{Q}\right) \leq P} E\left(\log \left|I_{N_{R}}+\frac{\left(A_{2} \mathbf{H}_{\mathbf{w}}+A_{1} \Sigma_{\mu}\right) \Sigma_{Q}\left(A_{2} \mathbf{H}_{\mathbf{w}}+A_{1} \Sigma_{\mu}\right)^{H}}{\sigma^{2}}\right|\right) .
$$

Let $\mathrm{L}=A_{1} \Sigma_{\mu}+A_{2} \mathbf{H}_{\mathbf{w}}$, and substituting $L$ into the above formula yields

$$
\begin{aligned}
C & =\max _{\Sigma_{Q}: \operatorname{Tr}\left(\Sigma_{Q}\right) \leq P} E\left(\log \left|I_{N_{R}}+\frac{L \Sigma_{Q} L^{H}}{\sigma^{2}}\right|\right) \\
& =\max _{\Sigma_{Q}: \operatorname{Tr}\left(\Sigma_{Q}\right) \leq P} E\left(\log \left|I_{N_{R}}+\sum_{i=1}^{N_{T}} \frac{L_{\bullet} L_{\bullet i}^{H} \lambda_{i}^{Q}}{\sigma^{2}}\right|\right),
\end{aligned}
$$

Where $L_{. i}$ represents the $i$ th column of the matrix $L$, $\lambda_{i}^{Q}$ is the $i$ th entry along the diagonal of $\Sigma_{Q}$. Now, we impose a constraint of being unit rank on the covariance matrix $Q$ to derive the condition of beamforming optimality. Without loss of generality, assume that

$$
\begin{aligned}
\Sigma_{Q} & =\operatorname{diag}\left\{\lambda_{1}^{Q}, \lambda_{2}^{Q}, \ldots, \lambda_{N_{T}}^{Q}\right\} \\
& =\operatorname{diag}\left\{P-p, \beta_{2} p, \beta_{3} p, \ldots, \beta_{N_{T}} p\right\}
\end{aligned}
$$

where the sum of $\left\{\beta_{i}, i=2, \ldots, N_{T}\right\}$ is 1. Formula (11) can be further expressed as

$$
C=\max _{\Sigma_{Q}: T r\left(\Sigma_{Q}\right) \leq P} E\left(\log \left|I_{N_{R}}+(P-p) \frac{L_{\bullet} L_{* 1}^{H}}{\sigma^{2}}+\sum_{i=2}^{N_{T}} \beta_{i} p \frac{L_{\bullet i} L_{0_{i}}^{H}}{\sigma^{2}}\right|\right) .
$$

As the function $C$ is concave with respect to $p$, the necessary condition for beamforming optimality is $\partial C /$ $\left.\partial p\right|_{p=o} \leq 0$. Differentiating $C(p)$ with respect to $p$ and noting that the derivative of the function $\log |A+x B|$ at $x=0$ is $\operatorname{tr}\left(A^{-1} B\right)$, we have

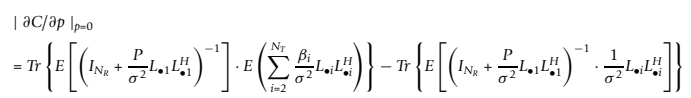

The second term on the right-hand side of (14) can be further written as

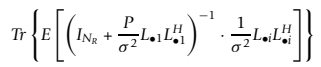

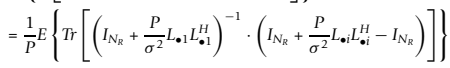

$$
\begin{aligned}
& =\frac{1}{P} E\left\{T_{r}\left[I_{N_{R}}-\left(I_{N_{R}}+\frac{P}{\sigma^{2}} L_{0.1} L_{01}^{H}\right)^{-1}\right]\right\}=\frac{1}{P}\left\{N_{R}-\left(N_{R}-1+E\left(\frac{1}{1+P / \sigma^{2}\left\|L_{0} \mid\right\|^{2}}\right)\right)\right\} \\
& =\frac{1}{P}\left[1-E\left(\frac{1}{1+P / \sigma^{2} \| L_{0}||^{2}}\right)\right] \text {. }
\end{aligned}
$$

Proceeding with the derivation of (14), we have

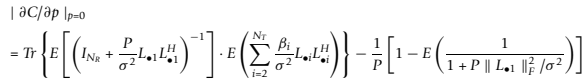

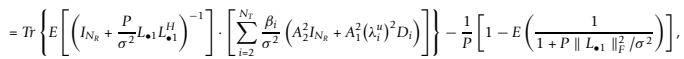

where $A_{1}$ and $A_{2}$ are defined in (2), $\lambda_{i}^{\mu}$ is the $i$ th diagonal element of the matrix $\Sigma_{\mu}, D_{i}=\operatorname{diag}(0, \ldots, 1, \ldots, 0)$ is an $N_{R}$ by $N_{R}$ matrix with the $i$ th diagonal element being 1 , $\|\cdot\|_{F}$ stands for Frobenius norm. Since the sum of $\left\{\beta_{j}\right\}$ is 1 , we have

$$
\begin{aligned}
& |\partial C / \partial p|=0
\end{aligned}
$$

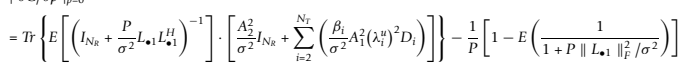

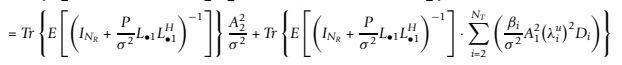

$$
\begin{aligned}
& -\frac{1}{P}\left[1-E\left(\frac{1}{1+P\left\|L_{L}\right\| \|_{e}^{2} / \sigma^{2}}\right)\right]
\end{aligned}
$$

To further derive the above formula, we consider the following matrix inversion lemma [14].

Lemma 1: For the matrix $B=A+\mathbf{x y}^{H}$, where $\mathbf{x}$ and $\mathbf{y}$ are two vectors, the inversion of $B$ is $B^{-1}=A^{-1}-\frac{A^{-1} \mathbf{x y}^{H} A^{-1}}{1+\mathbf{y}^{H} A^{-1} \mathbf{x}}$.

Proceeding with the derivation of (17), the second term on the right-hand side of (17) can be further expressed as

$$
\begin{aligned}
& \operatorname{Tr}\left\{E\left[\left(I_{N_{R}}+\frac{P}{\sigma^{2}} L_{\bullet} L_{\bullet}^{H}\right)^{-1}\right] \cdot \sum_{i=2}^{N_{T}}\left(\frac{\beta_{i}}{\sigma^{2}} A_{1}^{2}\left(\lambda_{i}^{u}\right)^{2} D_{i}\right)\right\} \\
& =\frac{A_{1}^{2}}{\sigma^{2}} \sum_{i=2}^{N_{T}}\left\{\beta_{i}\left(\lambda_{i}^{u}\right)^{2} \cdot \operatorname{Tr}\left[E\left[\left(I_{N_{R}}+\frac{P}{\sigma^{2}} L_{\bullet} L_{\mathbf{\bullet}_{1}}^{H}\right)^{-1}\right] \cdot D_{i}\right]\right\} \\
& =\frac{A_{1}^{2}}{\sigma^{2}} \sum_{i=2}^{N_{T}}\left\{\beta_{i}\left(\lambda_{i}^{u}\right)^{2} \cdot \operatorname{Tr}\left[E\left(I_{N_{R}}-\frac{\frac{P}{\sigma^{2}} L_{\bullet}^{H} L_{\bullet}}{1+\frac{P}{\sigma^{2}}\left\|L_{\bullet 1}\right\|_{F}^{2}}\right) \cdot D_{i}\right]\right\} \text {. }
\end{aligned}
$$

Since $D_{i}=\operatorname{diag}(0, \ldots, 1, \ldots, 0)$ with the $i$ th diagonal element being 1 , we have

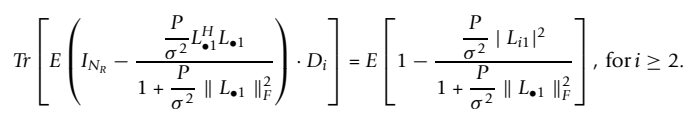

In consideration of $L_{\bullet}=A_{1} \lambda_{1}^{\mu}+A_{2}\left(\mathbf{H}_{\mathbf{w}}\right)_{\bullet}$, where $\left(\mathbf{H}_{\mathbf{w}}\right)_{\bullet 1}$ stands for the first column of the matrix $\mathbf{H}_{\mathbf{w}}$, it is found that

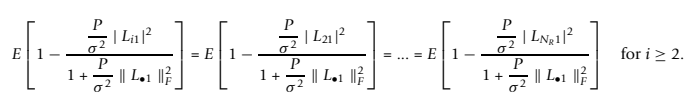

Therefore, without loss of generality, (18) can be further written as 


$$
\begin{aligned}
& \operatorname{Tr}\left\{E\left[\left(I_{N_{R}}+\frac{P}{\sigma^{2}} L_{\bullet 1} L_{\bullet 1}^{H}\right)^{-1}\right] \cdot \sum_{i=2}^{N_{T}}\left(\frac{\beta_{i}}{\sigma^{2}} A_{1}^{2}\left(\lambda_{i}^{u}\right)^{2} D_{i}\right)\right\} \\
& =\frac{A_{1}^{2}}{\sigma^{2}} \sum_{i=2}^{N_{T}}\left\{\beta_{i}\left(\lambda_{i}^{u}\right)^{2} E\left[1-\frac{\frac{P}{\sigma^{2}}\left|L_{21}\right|^{2}}{1+\frac{P}{\sigma^{2}}\left\|L_{\bullet 1}\right\|_{F}^{2}}\right]\right\} \\
& =\frac{A_{1}^{2}}{\sigma^{2}} \sum_{i=2}^{N_{T}}\left(\beta_{i}\left(\lambda_{i}^{u}\right)^{2}\right) \cdot E\left[1-\frac{\frac{P}{\sigma^{2}}\left|L_{21}\right|^{2}}{1+\frac{P}{\sigma^{2}}\left\|L_{\bullet 1}\right\|_{F}^{2}}\right]
\end{aligned}
$$

where $L_{i j}$ is the $(i, j)$ th entry of the matrix $L$. Since the condition $\partial C /\left.\partial p\right|_{p=0} \leq 0$ is satisfied, the maximum value of (16) is less than or equal to 0 . When $\beta_{2}=1, \beta_{j}$ $=0$ for $j>2$, the formula (21) achieves the maximum value and so does (16). Thus, manipulating (16), (17), and (21), the necessary condition for beamforming optimality is given by

$$
F\left(P, \sigma^{2}, N_{T}, N_{R}, \Sigma_{\mu}, K\right) \leq 0,
$$

where

$$
\begin{aligned}
& F\left(P, \sigma^{2}, N_{T}, N_{R}, \Sigma_{\mu}, K\right) \\
& =\frac{A_{2}^{2} P\left(N_{R}-1\right)}{\sigma^{2}}-1+\left(1+\frac{A_{2}^{2} P}{\sigma^{2}}\right) E\left(\frac{1}{1+P / \sigma^{2}\left\|L_{\bullet}\right\|_{F}^{2}}\right)+\frac{P}{\sigma^{2}} A_{1}^{2}\left(\lambda_{2}^{u}\right)^{2}\left[1-E\left(\frac{P / \sigma^{2}\left|L_{21}\right|^{2}}{1+P / \sigma^{2}\left\|L_{\bullet 1}\right\|_{F}^{2}}\right)\right]
\end{aligned}
$$

And the function $F(\cdot)$ is referred to as the beamforming function.

Finally, the necessary condition expressed by (22) is also the sufficient condition because the second derivative of $C$ $(p)$ is equal to or less than 0 , i.e., $\frac{\partial^{2} C(p)}{\partial p^{2}} \leq 0$. The sufficiency proof is the same as that in [6]. Thus, it is concluded that the necessary and sufficient condition for beamforming optimality is given by the inequality (22). When the condition (22) is satisfied, we can adopt the beamforming strategy at the transmitter to achieve the maximum capacity of MIMO systems under mean feedback. The beamforming strategy includes three steps:

Step 1: The transmitter obtains the mean feedback $\mathbf{H}_{\boldsymbol{\mu}}$ from the receiver and performs SVD to retrieve the eigenvector $V_{\mu}$, as in Equation 7.

Step 2: The optimal power allocation matrix is set as $\Sigma_{Q, B F}=|\operatorname{diag}\{P, 0, \ldots, 0\}|_{N_{T} \times N_{T}}$.

Step 3: Assuming the original user data $\mathbf{x}^{\prime}=\left\{x_{i}^{\prime}, i=1,2, \ldots, N_{T}\right\}$ is coded by i.i.d Gaussian code and therefore each data stream $x_{i}^{\prime}$ is i.i.d Gaussian distributed. Then, the transmitter performs linear transformation to obtain the transmitted signal $\mathbf{x}$, i.e., $\mathbf{x}=\Sigma_{Q, \mathrm{BF}}^{1 / 2} V_{\mu} \mathbf{x}^{\prime}$

\subsection{Numerical evaluation for beamforming optimality condition}

Let $z_{1}\left|L_{21}\right|^{2}$ and $z_{2}=\left\|L_{\bullet 1}\right\|_{F}^{2}$, the probability density function (PDF) of $z_{1}$ is given by

$$
f_{z_{1}}(x)=\left\{\begin{array}{l}
(K+1) e^{-(K+1) x}, \text { for } x \geq 0 ; \\
0, \quad \text { else. }
\end{array}\right.
$$

Let $z_{3}=z_{2}-z_{1}$, then the PDF of $z_{3}$ can be obtained from the transform of the PDF of a non-central Chisquared distributed random variable and it is given by

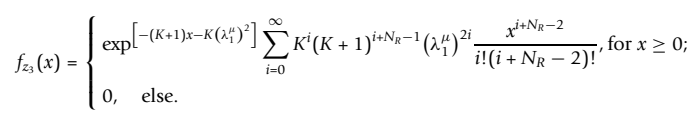

As the $z_{3}$ is independent of $z_{1}$ and the joint PDF of $\left(z_{1}, z_{3}\right)$ is given by

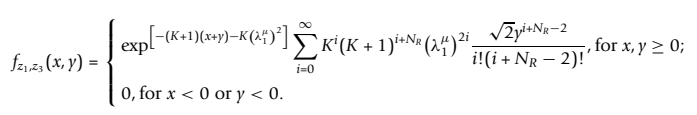

where $\Gamma(\cdot)$ is the Gamma function. As the Jacobian determinant of $\left(z_{1}, z_{3}\right)$ with respect to $\left(z_{1}, z_{2}\right)$ is 1 , the joint PDF of $\left(z_{1}, z_{2}\right)$ is given by

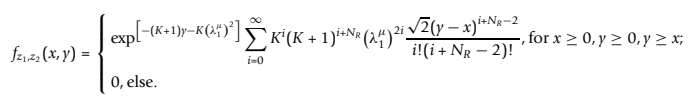

Therefore, with formulas (25) and (27), the necessary condition for beamforming optimality given by (22) can be evaluated by numerical method.

\section{Ergodic capacities for beamforming and optimum strategies}

This section presents the ergodic capacities for beamforming and optimum strategies. First, when the condition (22) for optimality of beamforming is satisfied, we can adopt the beamforming strategy to achieve the maximum capacity of MIMO systems. Substituting the beamforming transmitter power allocation matrix into (10), we have the capacity for beamforming, $C_{\mathrm{BF}}$, given by

$$
C_{\mathrm{BF}}=E\left(\log \left|I_{N_{R}}+\frac{\left(A_{2} \mathbf{H}_{\mathbf{w}}+A_{1} \Sigma_{\mu}\right) \Sigma_{Q, \mathrm{BF}}\left(A_{2} \mathbf{H}_{\mathbf{w}}+A_{1} \Sigma_{\mu}\right)^{H}}{\sigma^{2}}\right|\right),
$$

where $\Sigma_{Q, B F}=|\operatorname{diag}\{P, 0, \ldots, 0\}|_{N_{T} \times N_{T}}$. Second, the optimum strategy means that the optimal transmitter covariance matrix of maximizing the system capacity of (10) is determined by numerical optimization method. A few algorithms have been proposed to search the optimal covariance matrix $[15,16]$. Specifically, the optimal transmission strategy also includes three steps. Steps 1 and 3 are the same as that of beamforming strategy. Merely, the optimal power allocation matrix $\Sigma_{Q, O P T}$ is acquired by numerical searching method in Step 2. Once $\Sigma_{Q, O P T}$ is found, the ergodic capacity for optimum strategy is obtained by substituting it into formula (10).

\section{Numerical and simulation results}

Both numerical method and computer simulation have been deployed to examine the beamforming optimality 
condition and ergodic capacity. The condition of beamforming optimality expressed by (22) is evaluated by numerical method. The ergodic capacity for beamforming strategy given by (28) is evaluated by computer simulation. For the sake of comparison, we also present the ergodic capacities for equal power allocation and optimum strategy, respectively. Table 1 lists the singular values of channel mean matrix for different antenna pairs.

\subsection{The effects of Ricean factor and antenna pair on the beamforming function $f$}

Figure 1 shows the beamforming function $F$ versus Ricean factor $K$, for different values of SNRs. The antenna pair is $(2,2)$ and the corresponding matrix $\Sigma_{\mu}$ is given by Table 1 . Observe that $F$ decreases when $K$ increases from -10 to $20 \mathrm{~dB}$ at a fixed SNR. For SNR = $5 \mathrm{~dB}, F$ decreases from 2.76 to 1.68 when $K$ increases from -10 to $5 \mathrm{~dB}$. Further increasing $K$ only marginally decreases $F$. Similar behaviors can be found for the other two cases. Increase of SNR results in increase of $F$. It is found that the beamforming optimality condition is satisfied as $F \leq 0$ when $K>-2.6 \mathrm{~dB}$ for the case of $\mathrm{SNR}=-5 \mathrm{~dB}$. It does not hold for the other two cases.

Figure 2 depicts the beamforming function $F$ for several antenna pairs. Observe that $F$ increases with increasing SNR. For $4 \times 4$ system, $F$ increases rapidly when SNR is larger than $0 \mathrm{~dB}$. Similar behaviors can be found for $2 \times 2$ and $4 \times 2$ systems. However, for $2 \times 1$ system, the beamforming function $F$ remains around 0 for SNR ranging from -10 to $10 \mathrm{~dB}$. The SNR thresholds for $F=0$ are approximately equal to $-3,0.2$, and $-4.5 \mathrm{~dB}$ for $2 \times 2,4 \times 2$, and $4 \times 4$ systems, respectively. Thus, it is observed that the beamforming optimality condition tends to hold for relatively low SNR and small number of receiver antennas.

\subsection{Capacity comparisons among several transmission strategies}

Figure 3 depicts the $2 \times 1$ system ergodic capacities of the beamforming, the optimum strategy, and the equal power allocation, respectively. Observe that the capacity for optimum strategy has about $0.8 \mathrm{~dB}$ gain over that of equal power allocation when achieving the same ergodic capacity. The capacity for beamforming strategy is nearly the same as that of optimum strategy for SNR ranging from -5 to $20 \mathrm{~dB}$. Note that the beamforming optimality condition holds for $\mathrm{SNR} \in[-5$ $\mathrm{dB}, 20 \mathrm{~dB}]$.
Figure 4 depicts the ergodic capacities of the three cases for $2 \times 2$ system. Observe that the capacity for beamforming strategy is nearly the same as that of optimum strategy for SNR ranging from -15 to $0 \mathrm{~dB}$. However, the beamforming strategy is inferior to the optimum strategy when SNR is larger than $0 \mathrm{~dB}$. The equal power allocation is inferior to the optimum strategy when $\mathrm{SNR}<0 \mathrm{~dB}$. However, it is marginally the same as that of the optimum strategy at $\mathrm{SNR} \geq 0 \mathrm{~dB}$. Therefore, it is clear that the transmitter can adopt the beamforming strategy for $\mathrm{SNR}<0 \mathrm{~dB}$ and the equal power allocation method for $\mathrm{SNR} \geq 0 \mathrm{~dB}$. Note that the beamforming optimality condition only holds for SNR < $-3 \mathrm{~dB}$. But, the simulation results show that beamforming strategy is nearly optimal for SNR $<0 \mathrm{~dB}$. Meanwhile, the beamforming function $F$ is equal to 0.32 at $\mathrm{SNR}=0 \mathrm{~dB}$.

Figure 5 compares the $4 \times 2$ system ergodic capacities for the beamforming strategy, the optimum strategy, and the equal power allocation, respectively. Observe that the capacity for beamforming strategy is almost the same as that of optimum one for SNR $<2 \mathrm{~dB}$. The equal power allocation is much inferior to both the beamforming and optimum strategies. Note that beamforming function $F$ is about equal to 0 at $\mathrm{SNR}=0.2 \mathrm{~dB}$ and 0.33 at $\mathrm{SNR}=2 \mathrm{~dB}$.

As in Figure 6, it is clear that the transmitter can adopt the beamforming strategy for $\mathrm{SNR}<-3 \mathrm{~dB}$ and the equal power allocation method for SNR $>0 \mathrm{~dB}$. Note that beamforming function $F$ is equal to 0.22 at SNR $=-3 \mathrm{~dB}$. Combining the results with those of Figures 3 and 4, it is found that the beamforming strategy achieves nearly the same capacity with that of the optimum strategy, if $F$ is approximately less than 0.3 . So, it is concluded that the beamforming strategy can be adopted to achieve the same capacity with the optimum strategy on a relatively relaxed constraint of $F \leq 0.3$.

\section{Conclusions}

The beamforming optimality condition for MIMO systems under channel mean feedback has been derived. The rank of channel mean matrix, $r$, is not limited to one and satisfies $1 \leq r \leq \min \left(N_{T}, N_{R}\right)$. The condition has also been examined using a few examples with different channel mean matrices and antenna pairs. Numerical results show that the beamforming optimality condition tends to hold for relatively low SNR and small number of receiver antennas. Three transmission strategies including the beamforming strategy, the optimum

Table 1 The channel mean matrix, $\Sigma_{\mu \nu}$ in (7) for different antenna pairs

\begin{tabular}{lllll}
\hline Antenna pair & $\mathbf{( 2 ,} \mathbf{1})$ & $\mathbf{( 2 ,} \mathbf{2})$ & $\mathbf{( 4 ,} \mathbf{2})$ & $\mathbf{( 4 ,}, \mathbf{4})$ \\
\hline$\Sigma_{\mu}$ & $\lambda_{1 \mu}=1.414$ & $\left(\boldsymbol{\lambda}_{1 \mu}, \lambda_{2 \mu}\right)=(1.78,0.9)$ & $\left(\boldsymbol{\lambda}_{1 \mu}, \lambda_{2 \mu}\right)=(2.74,0.69)$ & $\left(\boldsymbol{\lambda}_{1 \mu}, \lambda_{2 \mu}, \lambda_{3 \mu}, \lambda_{4 \mu}\right)=(3.66,0.92,0.92,0.92)$ \\
\hline
\end{tabular}




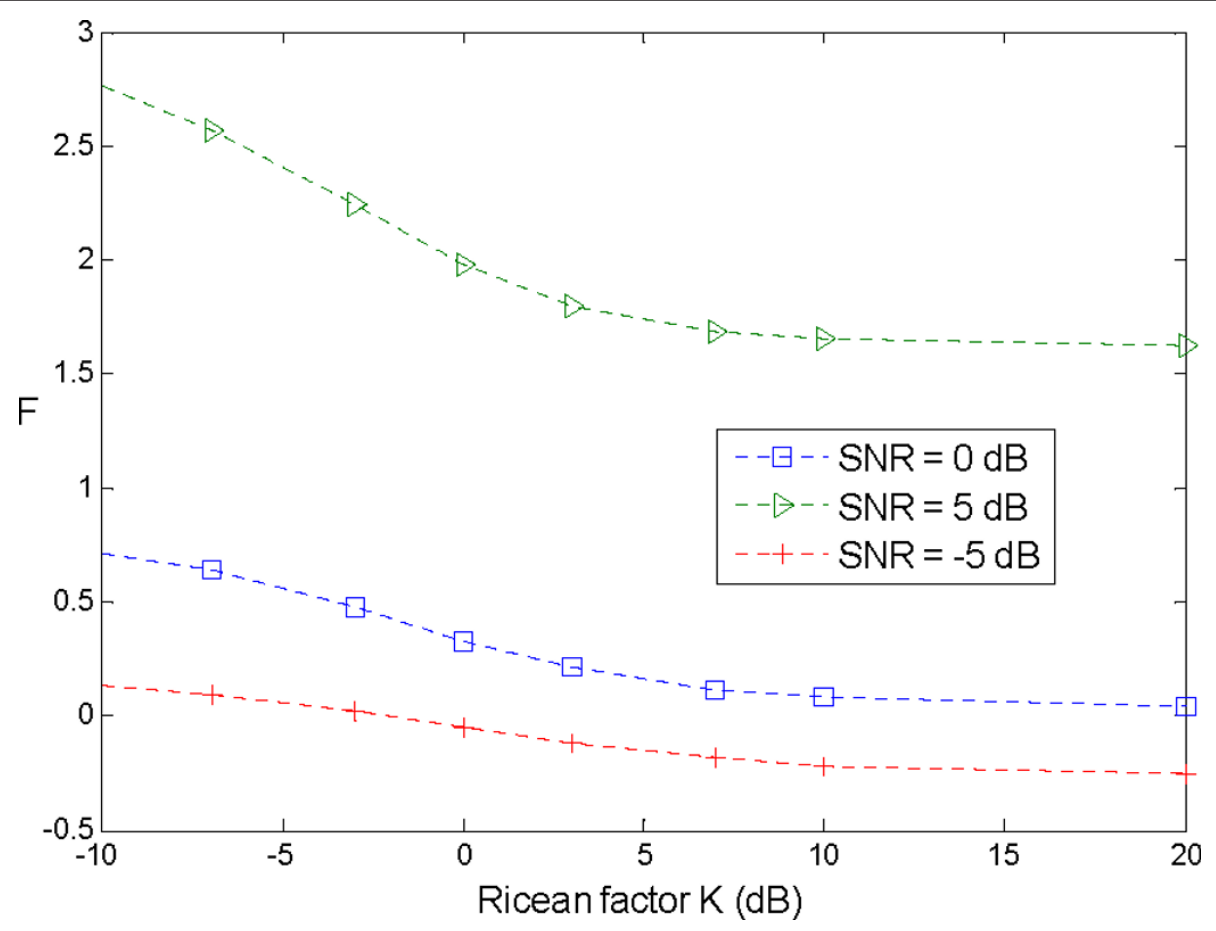

Figure 1 The beamforming function $F$ versus Ricean factor $K$ for $S N R=-5 d B, 0 d B$, and $5 \mathrm{~dB}$, respectively.

strategy, and the equal power allocation method, have been evaluated by computer simulation. Simulation results imply that the proposed beamforming strategy can achieve nearly the same capacity as that of the optimum strategy on a relatively relaxed constraint of $F \leq$ 0.3 .

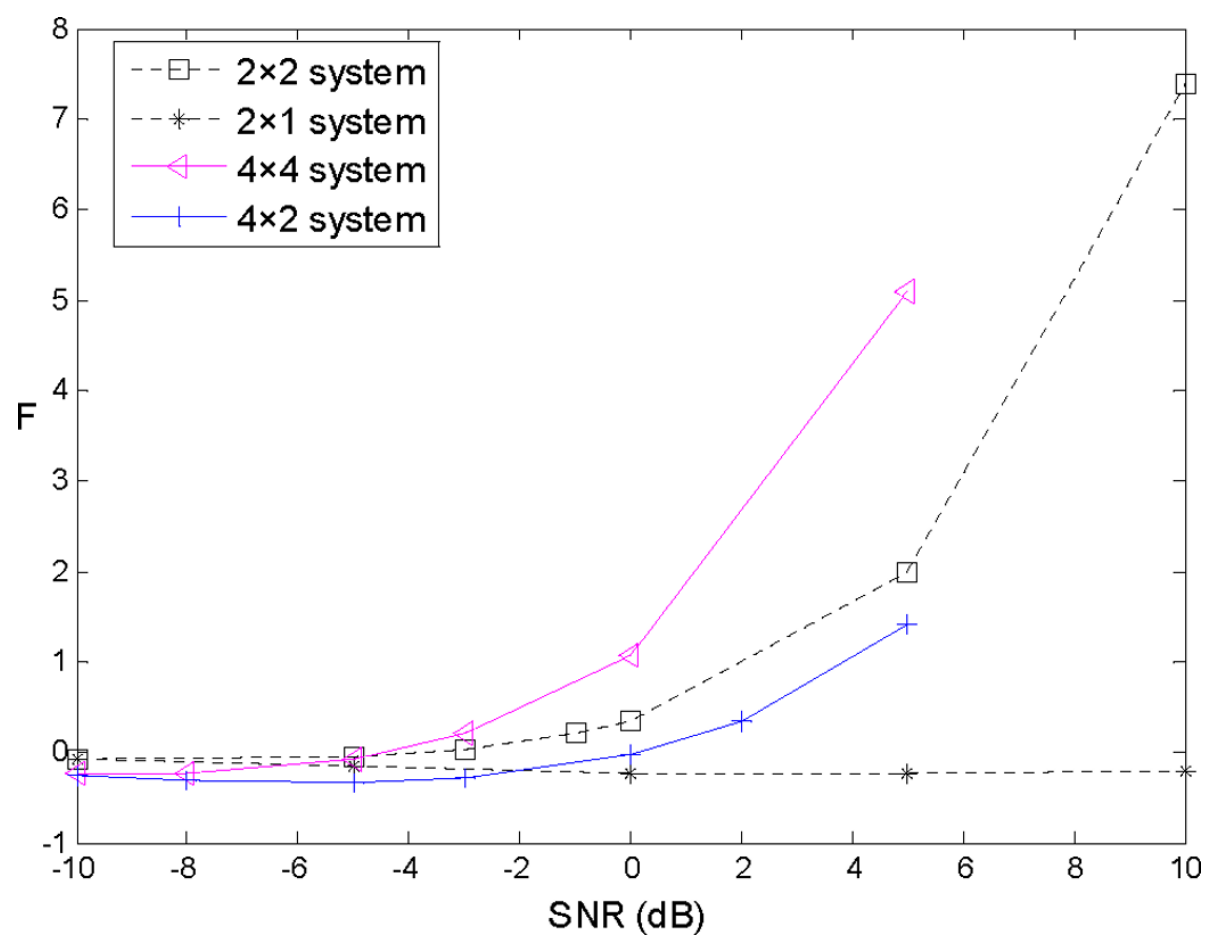

Figure 2 The beamforming function $F$ versus SNR, for different antenna pairs. 


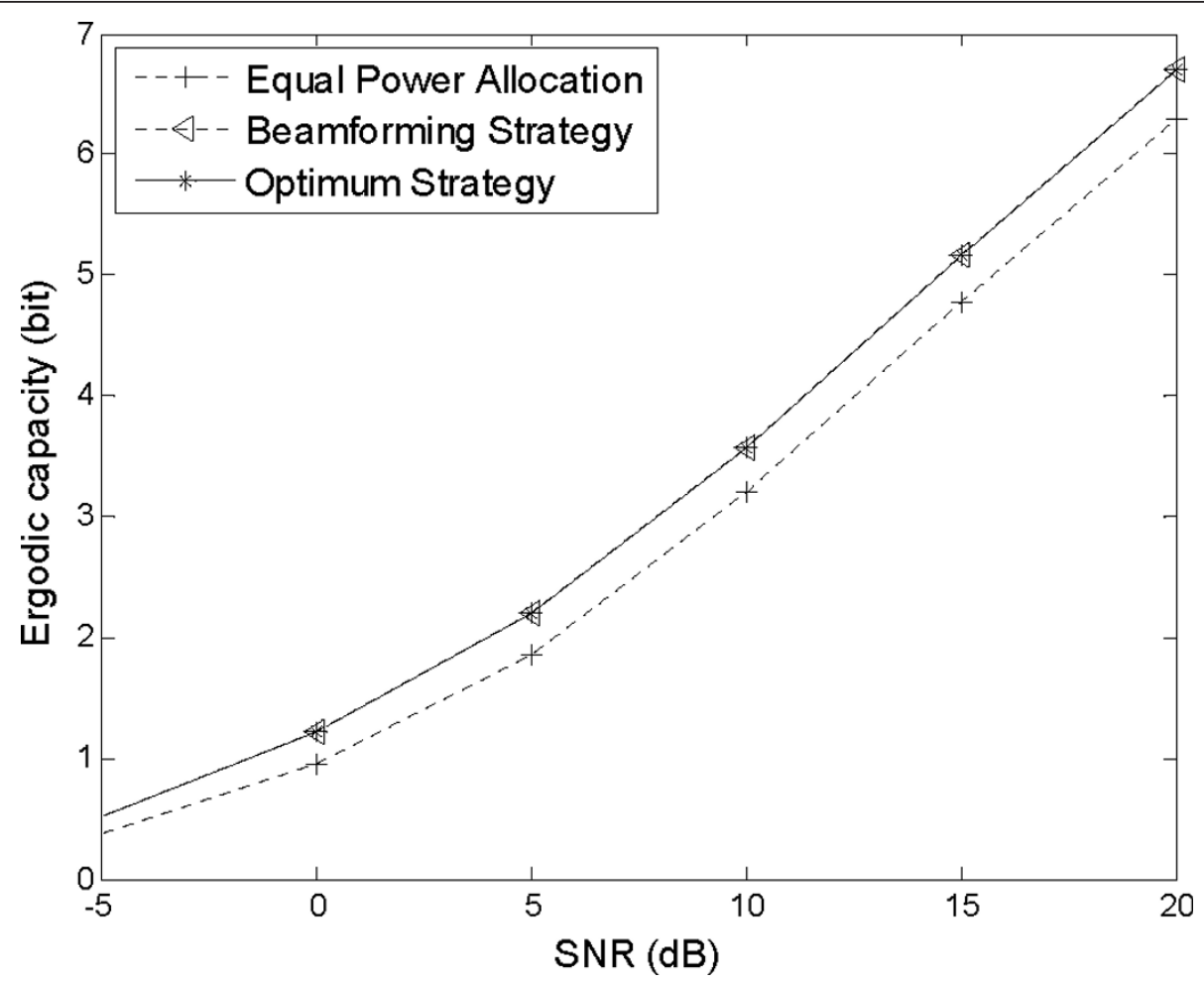

Figure 3 The $2 \times 1$ system ergodic capacity versus SNR for the beamforming strategy, the optimum strategy, and the equal power allocation, respectively.

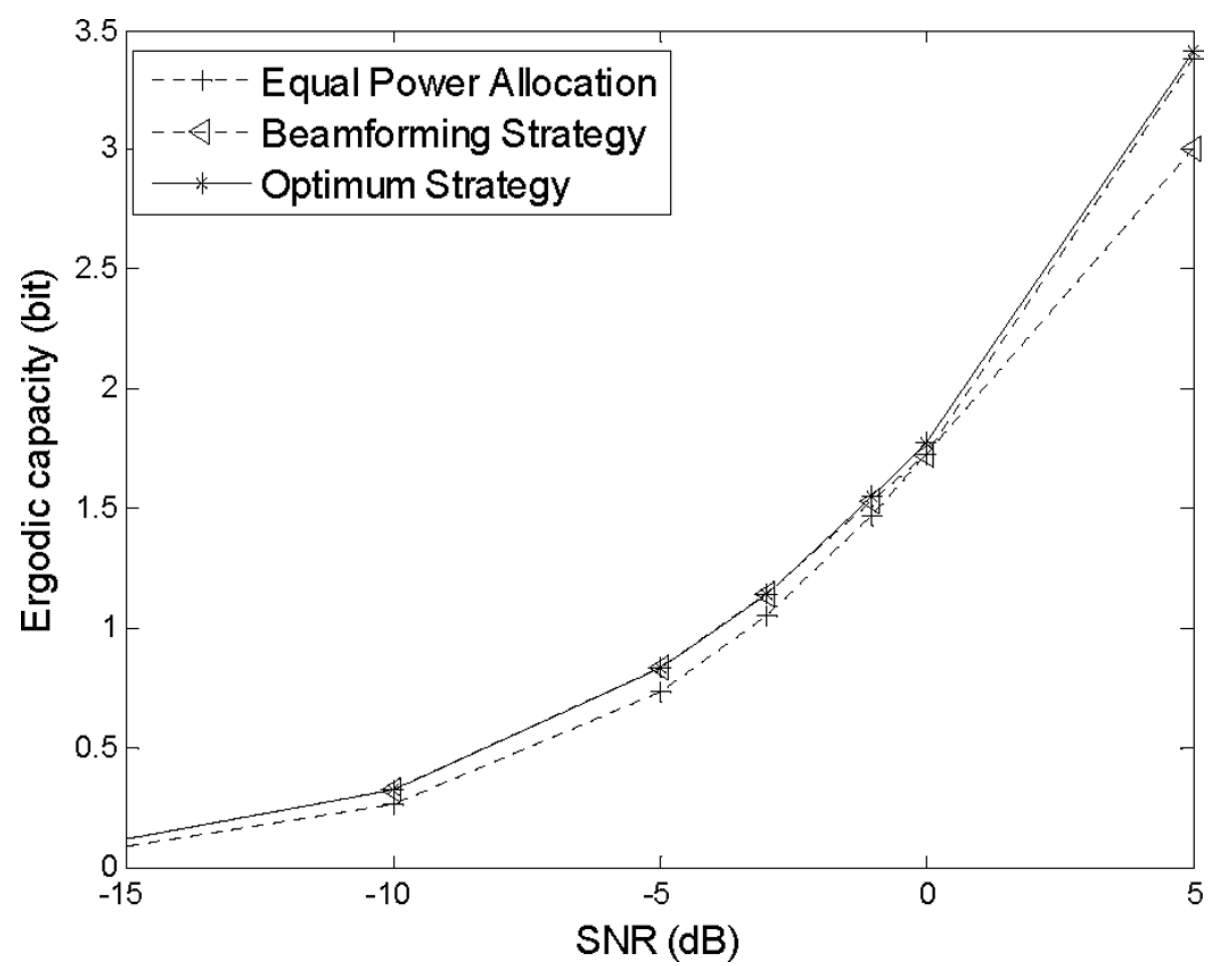

Figure 4 The $2 \times 2$ system ergodic capacity versus SNR for the beamforming strategy, the optimum strategy, and the equal power allocation, respectively. 


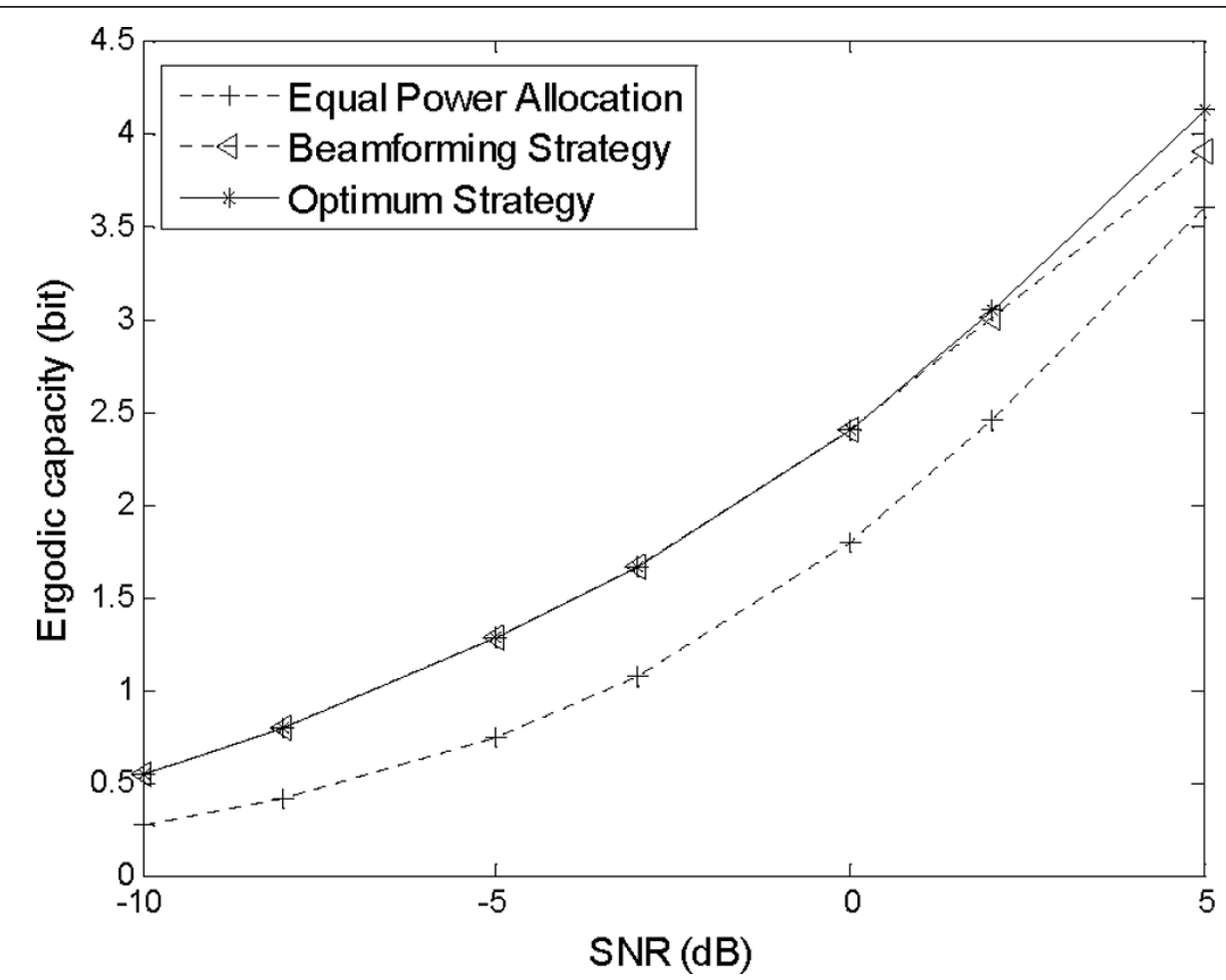

Figure 5 The $4 \times 2$ system ergodic capacity versus SNR for the beamforming strategy, the optimum strategy, and the equal power allocation, respectively.

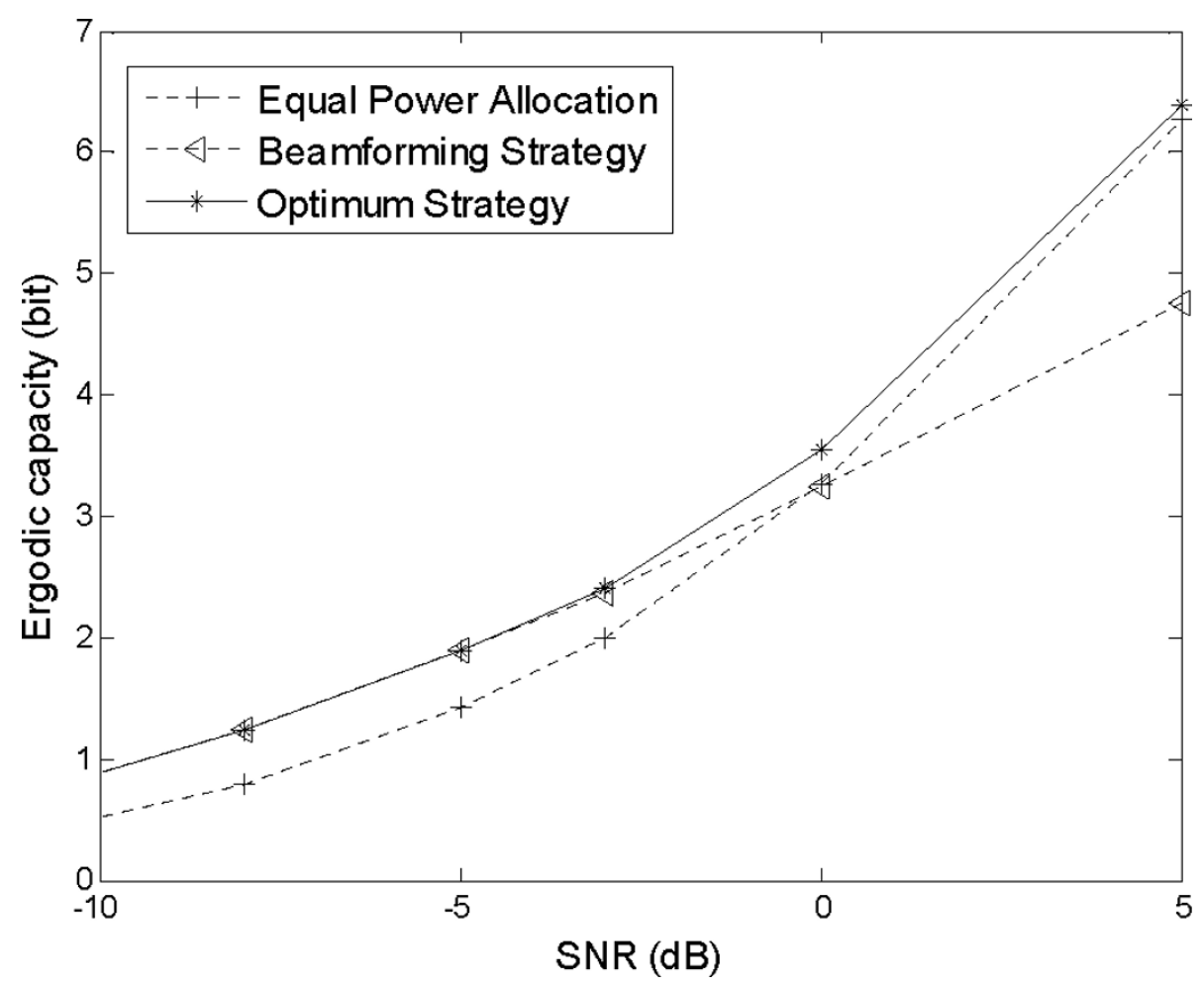

Figure 6 The $4 \times 4$ system ergodic capacity versus SNR for the beamforming strategy, the optimum strategy, and the equal power allocation, respectively. 


\section{Abbreviations}

CSI: channel state information; MIMO: multiple-input multiple output; PDF: probability density function; SNR: signal-to-noise ratio; SVD: singular value decomposition.

\section{Acknowledgements}

This study was supported by the Scientific Research Foundation of Shantou University of China (Grant no. NTF11004). In addition, the authors would like to thank the anonymous reviewers for their beneficial comments on this manuscript.

\section{Author details}

'Department of Electronic Engineering, Shantou University, Shantou, China ${ }^{2}$ Institute of Industrial Science, The University of Tokyo, Tokyo, Japan ${ }^{3}$ Department of Electronic and Electrical Engineering, The University of Hong Kong, Pokfulam, Hong Kong

\section{Competing interests}

The authors declare that they have no competing interests.

Received: 24 June 2011 Accepted: 10 November 2011 Published: 10 November 2011

\section{References}

1. 3GPP TS36.913 V9.0.0: Requirements for further advancements for E-UTRA (LTE-Advanced) (December 2009)

2. IE Telatar, Capacity of Multi-Antenna Gaussian Channels (AT\&T Bell Laboratories, 1995), BL0112170-950615-07TM

3. GJ Foschini, MJ Gans, On the limits of wireless communications in a fading environment when using multiple antennas. Wirel Personal Commun. 6(3), 315-335 (1998)

4. E Visotsky, U Madhow, Space-time transmit precoding with imperfect feedback. IEEE Trans Inf Theory 47(6), 2634-2639 (2001)

5. A Narula, MJ Lopez, MD Trott, GW Wornell, Efficient use of side information in multiple-antenna data transmission over fading channels. IEEE J Sel Areas Commun. 16(8), 1423-1436 (1998). doi:10.1109/49.730451

6. SA Jafar, A Goldsmith, Transmitter optimization and optimality of beamforming for multiple antenna systems. IEEE Trans Wirel Commun. 3(4), 1165-1175 (2004). doi:10.1109/TWC.2004.830822

7. JC Roh, BD Rao, Multiple antenna channels with partial channel state information at the transmitter. IEEE Trans Wirel Commun. 3(2), 677-688 (2004). doi:10.1109/TWC.2003.821144

8. EA Jorswieck, R Mochaourab, M Mittelbach, Effective capacity maximization in multi-antenna channels with covariance feedback. IEEE Trans Wirel Commun. 9(10), 2988-2993 (2010)

9. CB Chae, D Mazzarese, N Jindal, RW Heath, Coordinated beamforming with limited feedback in the MIMO broadcast channel. IEEE J Sel Areas Commun. 26(8), 1505-1515 (2008)

10. X Li, S Jin, X Gao, MR McKay, K-K Wong, Transmitter optimization and beamforming optimality conditions for double-scattering MIMO channels. IEEE Trans Wirel Commun. 7(9), 3647-3654 (2008)

11. S Srinivasa, SA Jafar, The optimality of transmit beamforming: a unified view. IEEE Trans Inf Theory 53(4), 1558-1564 (2007)

12. D Wu, R Negi, Effective capacity: a wireless link model for support of quality of service. IEEE Trans Wirel Commun. 2(4), 630-643 (2003)

13. S Venkatesan, SH Simon, RA Calenzuela, Capacity of a Gaussian MIMO channel with nonzero mean, in Proceedings of IEEE VTC, Orlando, 1767-1771 (2003)

14. TK Moon, WC Stirling, Mathematical Methods and Algorithms for Signal Processing (Prentice Hall, 2000)

15. LW Hanlen, AJ Grant, Optimal transmit covariance for MIMO channels with statistical transmitter side information, in Proceedings of IEEE ISIT, 1818-1822 (2005)

16. E Jorswieck, H Boche, Optimal transmission with imperfect channel state information at the transmit antenna array. Wirel Personal Commun. 27(1), 33-56 (2003). doi:10.1023/A:1026048118953

doi:10.1186/1687-1499-2011-160

Cite this article as: Zhou et al: Optimality of beamforming condition for multiple antenna systems with mean feedback. EURASIP Journal on Wireless Communications and Networking 2011 2011:160.

\section{Submit your manuscript to a SpringerOpen ${ }^{\mathcal{O}}$ journal and benefit from:}

- Convenient online submission

- Rigorous peer review

- Immediate publication on acceptance

- Open access: articles freely available online

- High visibility within the field

- Retaining the copyright to your article

Submit your next manuscript at $\gg$ springeropen.com 